\title{
UM ESTUDO DE CASO DA MOVIMENTAÇÃO DE NAVIOS DO PORTO DE ITAJAÍ A PARTIR DA TEORIA DAS FILAS E DA SIMULAÇÃO DISCRETA
}

\author{
Tatiana Cunha de Mello \\ Universidade Federal de Santa Catarina - Campus Universitário - Trindade - \\ Florianópolis - SC, Brasil. Caixa Postal 476 - CEP 88040-900 \\ tatianacdemello@gmail.com \\ Roberta Briesemeister \\ Universidade Federal de Santa Catarina - Campus Universitário - Trindade - \\ Florianópolis - SC, Brasil. Caixa Postal 476 - CEP 88040-900 \\ robries57@gmail.com \\ Ricardo Villarroel Dávalos \\ Universidade Federal de Santa Catarina - Campus Universitário - Trindade - \\ Florianópolis - SC, Brasil. Caixa Postal 476 - CEP 88040-900 \\ ricardo.davalos@ufsc.br
}

\begin{abstract}
Resumo
Sistemas prestadores de serviços frequentemente geram filas de usuários, ocasionam a perda de produtividade de todo o sistema, causam redução do nível de serviço prestado ao consumidor final e custos elevados, uma vez que estas geram atrasos por vezes desnecessários. A partir dos dados de movimentação de navios, fornecidos pelo Porto de Itajaí, foram modelados e analisados os tamanhos das filas e os tempos de espera das embarcações por disponibilidade de atendimento no porto, aplicando a Teoria das Filas e a Simulação Discreta para diferentes cenários de capacidade. Assim, avaliou-se o desempenho do sistema a partir do Software Arena e os dados foram modelados a partir da ferramenta Input Analyzer. Por fim, realizou-se uma análise comparativa da aplicação destas técnicas.
\end{abstract}

Palavras-Chaves: Modelagem; Simulação discreta; Teoria das filas.

\begin{abstract}
Service provider systems often generate queues of users. The queues lead to efficiency loss in the entire system, causing reduction in the service level provided to the end user and cost increase, since they generate unnecessary delays. From the vesselhandling data supplied by the Port of Itajaí, we analyzed the queues and the waiting times of vessels for availability of service at the port by applying system modeling and simulation of different scenarios of capacity. We used the Arena software and its Input Analyzer tool to evaluate the system performance. Finally, the simulation results were compared to the results previously obtained by applying the theory of queues.

Keywords: $\quad$ Modeling; Discrete Simulation; Theory of Queues.
\end{abstract}




\section{INTRODUÇÃO}

A expansão do comércio internacional está diretamente ligada à atividade portuária, uma vez que grande parte das mercadorias que circulam pelo mundo são transportadas em navios e movimentadas pelos portos. O complexo portuário brasileiro movimentou, em 2013, 931 milhões de toneladas de carga bruta, apresentando um crescimento de $2,9 \%$ em relação a 2012. Sozinho, o setor portuário é responsável por mais de $90 \%$ das exportações do País [13].

O transporte marítimo é um dos mais importantes modais de transporte. Atende cerca de $80 \%$ do volume de mercadorias comercializadas internacionalmente e é a única opção eficaz, em termos de custo, para o transporte de grandes volumes entre continentes [16].

Um moderno terminal de contêiner é um empreendimento industrial onde uma grande variedade de atividades acontece ao mesmo tempo. O principal propósito de toda esta atividade é transferir mercadorias em contêineres o mais rápido e eficientemente possível. O terminal de contêiner tem uma participação central do transporte internacional de mercadorias e é um elo essencial na cadeia do transporte. A eficiência com a qual o terminal executa sua função tem um impacto muito significativo na velocidade, uniformidade e custo do transporte de carga do exportador para o importador [17].

A entrada e saída de mercadorias do país é um processo bastante complexo no sentido de que se estas operações não forem executadas dentro de um processo altamente alinhado com armadores, agentes de cargas ou importadores e exportadores, a operação será extremamente onerada pelos impactos dos custos relacionados por eventuais atrasos que ocorram.

Com a finalidade de redução de custos e de possíveis filas, a atividade de programação de navios passa a ganhar mais importância no contexto da operação dos navios. A programação de navios lida com a alocação de um conjunto de cargas aos navios da rota que estão disponíveis, buscando minimizar os custos relacionados com a operação [3].

As instalações do Porto de Itajaí têm mais de $15.000 \mathrm{~m}^{2}$ de área coberta para estocagem de produtos e $38.000 \mathrm{~m}^{2}$ de área descoberta para armazenagem de contêineres. Os usuários do Porto de Itajaí têm a sua disposição, a qualquer momento, mais de 70 equipamentos, com capacidade de 1 a 37 toneladas para auxílio na carga e descarga de suas mercadorias [15].

O Porto de Itajaí, conta ainda com uma Estação Aduaneira de Interior (porto seco), totalmente alfandegada e sincronizada com o Porto, com $31.500 \mathrm{~m}^{2}$ para armazenagem coberta e pátios de armazenagem de contêineres com mais de $120.000 \mathrm{~m}^{2}$ de área. Sendo tradicionalmente um porto de carga geral, vem apresentando um grande crescimento nestes últimos anos [15].

Este trabalho tem como proposta avaliar melhorias para poder minimizar o tempo de espera dos navios no Porto de Itajaí, e consequentemente no sistema, possibilitando a adoção de políticas que tragam reais benefícios no desempenho do sistema melhorando sua eficiência.

\section{TEORIA DAS FILAS}

Um dos problemas encontrados em diversos sistemas de produção, particularmente em sistemas de serviços, tais como supermercados, bancos, postos de gasolina, atendimento em call centers, entre outros, são as filas, ou seja, tempo de 
espera para entrada e saída no sistema. Estas filas também aparecem em sistemas de transporte, como navios esperando para descarregar em portos, caminhões que levam cargas para serem posteriormente transportadas por outro modal, aviões para pouso e decolagem.

Um veículo que chega ao terminal para descarregamento de produtos e encontra uma posição de atendimento não ocupada pode se dirigir diretamente a essa doca para descarregamento. No entanto, muitas vezes as posições de descarga estão ocupadas e o veículo precisa entrar numa fila. Para o dimensionamento do número necessário de docas de recepção é necessário determinar o número de posições de descarga e o número esperado de veículos na fila, para isso adotando-se um nível de serviço adequado. O número de posições de descarga atuando em paralelo na doca de recepção é determinado pela aplicação de um modelo matemático de fila, ou por simulação.

As filas podem acarretar desde transtornos pessoais até prejuízos na produção, pagamento de altas multas e perda de futuros negócios. De maneira geral, um sistema com filas é qualquer processo aonde usuários oriundos de uma determinada população chegam para receber um serviço, e se for necessário, saindo do sistema assim que o serviço é completado. Essa espera acontece quando a demanda é maior do que a capacidade de atendimento oferecido, em termos de fluxo [7].

A literatura sobre modelos clássicos de filas inclui várias publicações, destacando-se Saaty (1961), Newell, (1971), Page (1972), Novaes (1975) e Fogliatti e Mattos (2007). Tais modelos têm como objetivo determinar e avaliar analiticamente medidas de desempenho, que expressam a produtividade e a operacionalidade desses processos, tais como número de elementos na fila, tempo de espera até o atendimento, tempo ocioso dos prestadores de serviço, etc. [6].

Um elemento importante nos modelos clássicos de filas é a notação de Kendall (Saaty, 1961; Novaes, 1975; Fogliatti e Mattos, 2007), que possibilita a classificação das filas de acordo com diversos fatores. Os principais fatores são: (a) o processo probabilístico de chegadas, (b) o processo probabilístico de atendimento, (c) o número de posições de atendimento em paralelo, (d) a capacidade física do sistema, (e) a

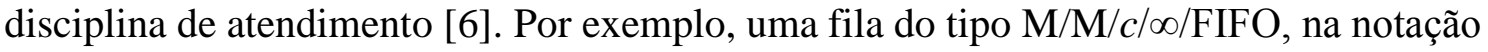
de Kendall, tem as seguintes características:

a) As chegadas dos elementos obedecem a um processo probabilístico de Poisson, que é representado pela letra $\mathrm{M}$;

b) Os tempos de atendimento nas instalações de serviço obedecem a uma distribuição exponencial negativa, também representada pela letra $\mathbf{M}$, pois a distribuição exponencial está intimamente ligada à distribuição de Poisson;

c) Existem $c$ posições de atendimento operando em paralelo;

d) A capacidade do sistema de filas é infinita $(\infty)$, podendo receber um número não restrito de unidades que desejam serem atendidas pelo sistema;

e) A ordem de atendimento da fila é FIFO (First In, First Out), conceito em inglês que significa que o atendimento é realizado na ordem de chegada.

A Figura 1 ilustra um sistema de fila genérico. 
Figura 1 - Representação de um sistema com fila.

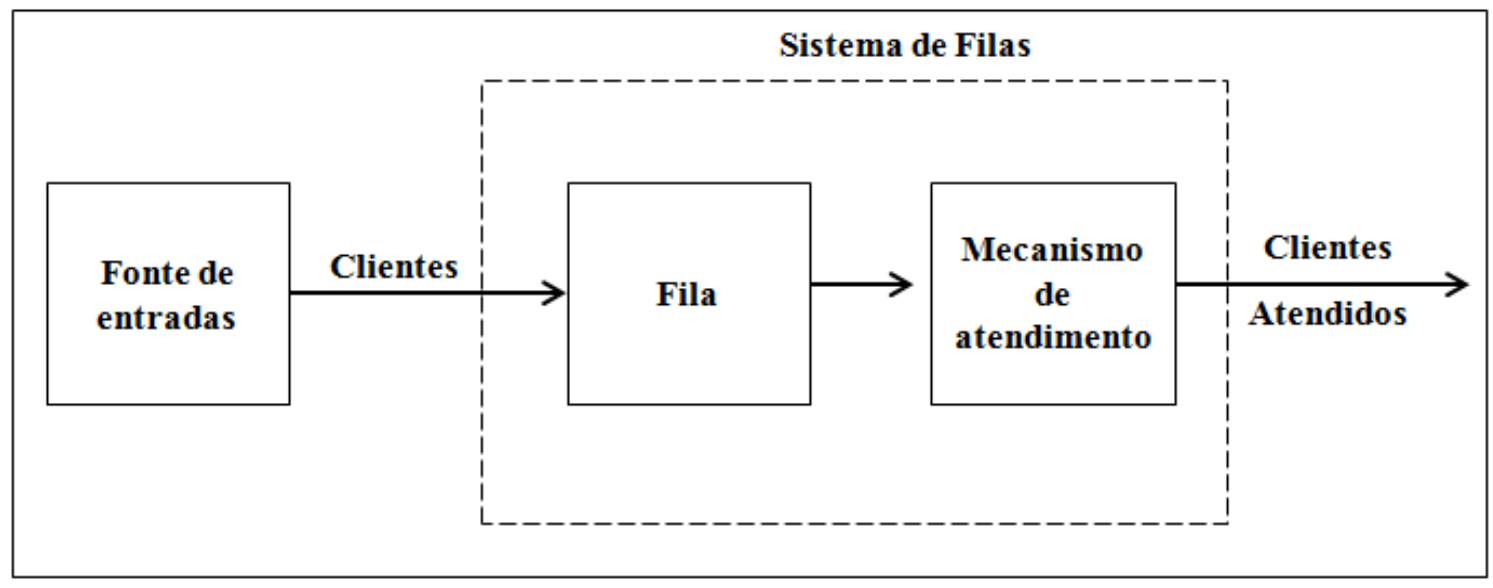

Fonte: Adaptado de [8].

O foco desta teoria é a avaliação de medidas de desempenho do sistema em função de uma dada configuração. As medidas de desempenho mais utilizadas são:

$>L$ : número médio de clientes no sistema;

$>L_{q}$ : número médio de clientes na fila;

$>W$ : tempo médio de espera no sistema de um cliente;

$>W_{q}$ : tempo médio de espera na fila por um cliente;

$>P_{i}$ : probabilidade de que o sistema esteja ocupado;

$>P_{0}$ : probabilidade de que o sistema esteja desocupado.

O modelo utilizado no presente estudo apresenta um número "c" de canais, uma capacidade de atendimento $\infty$ (infinita), a disciplina da fila é FIFO, ou seja, o atendimento ocorre de acordo com a ordem das chegadas dos usuários ao sistema (M/M/c /o/FIFO). A taxa de utilização é definida a partir da equação 1.

$$
\rho=\frac{\lambda}{c . \mu}
$$

Onde $\lambda$ e $\mu$, representam os ritmos de chegada e de atendimento, respectivamente.

Esse sistema de filas não apresenta fila infinita se $\rho<1$.

As medidas de desempenho para este modelo de filas podem ser determinadas a partir das fórmulas 2-7.

$$
P_{0}=\left[\frac{c^{c} \cdot \rho^{c+1}}{c !(1-\rho)}+\sum_{n=0}^{c} \frac{(c . \rho)^{n}}{n !}\right]^{-1}
$$




$$
\begin{aligned}
& P i=\left\{\begin{array}{l}
\frac{\left(c^{c} \cdot \rho\right)^{i}}{i !} . P_{0} \quad \text { se } i=1, \ldots, c \\
\frac{c^{c} \cdot \rho^{i}}{c !} \cdot P_{0} \text { se } i=c+1, \ldots . .
\end{array}\right. \\
& L=\lambda \cdot W \\
& L_{q}=\frac{c^{c} \cdot\left(\rho^{c+1}\right)}{c !(1-\rho)^{2}} P_{0} \\
& W_{q}=\frac{L_{q}}{\lambda} \\
& W=W_{q}+\frac{1}{\mu}
\end{aligned}
$$

Desta forma, a teoria das filas é uma aplicação da teoria das probabilidades e que estuda a formação de filas, através de desenvolvimentos matemáticos como os apresentados nas equações 2-7.

\section{SIMULAÇÃO DISCRETA}

A teoria das filas por sua natureza pode-se considerar como um método analítico que aborda o assunto por meio de formulas matemáticas e a simulação discreta é uma técnica que usando o computador procura montar um modelo que melhor represente o sistema em estudo [12].

A simulação discreta vem sendo cada vez mais utilizada em diferentes áreas de conhecimento, tendo em vista a crescente complexidade dos problemas e a maior disponibilidade de recursos computacionais. Utiliza modelos lógicos e matemáticos, além de recursos computacionais, visando emular o funcionamento dos mais variados tipos de operação ou processos do mundo real [11].

Define-se a simulação como o processo de projetar um modelo computacional de um sistema real e conduzir experimentos, com o propósito de entender seu comportamento e/ou avaliar estratégias para sua operação. Dentre as vantagens do uso dos modelos de simulação, destaca-se que estes permitem obter conclusões sobre sistemas sem construí-los e sem perturbá-los, se existentes [9].

Num mundo cada vez mais competitivo e globalizado, a simulação tornou-se uma ferramenta muito potente para o planejamento, projeto e controle de sistemas. É vista hoje por muitos engenheiros, administradores, projetistas e pela maioria dos estudiosos como uma metodologia indispensável para a solução de problemas [10]. 
Figura 2 - Representação esquema de um modelo de sistema

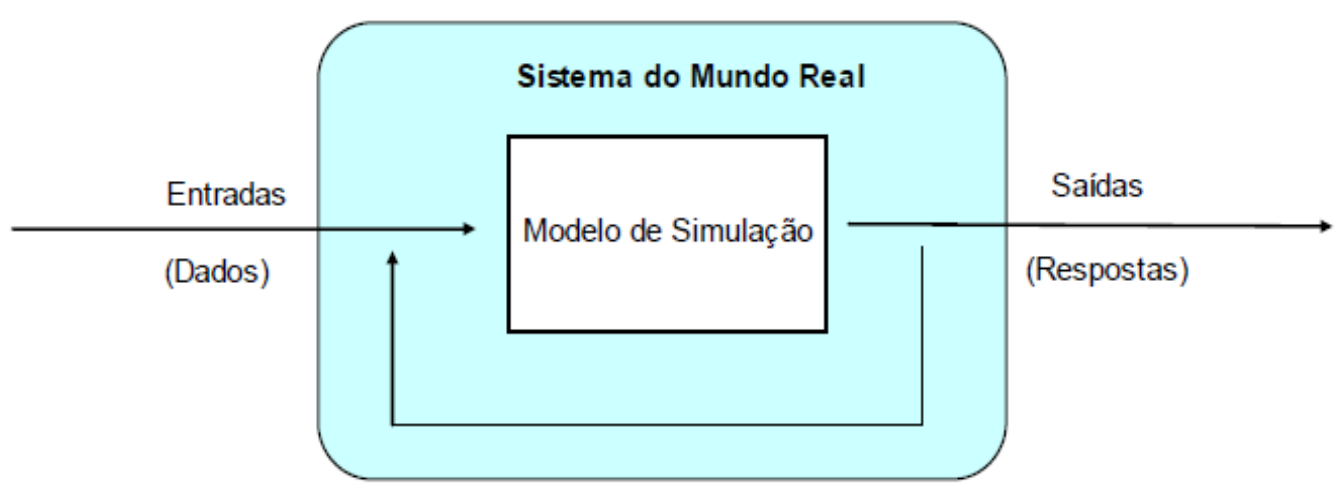

Fonte: Adaptado de [5].

As simulações reproduzem modelos para que sejam feitas análises e auxiliem nas tomadas de decisões ao contrário dos modelos de otimização que buscam uma solução ótima.

Os softwares de simulação podem ser de uso geral ou voltados para aplicações específicas, tais como transporte, manufatura, serviços, telecomunicações, reengenharia e outros. Os principais pacotes de simulação de uso geral são o Arena, ProModel, FlexSim, Micro Saint, Extend, AnyLogic, dentre outros [14].

Algumas características destes pacotes são o ambiente de trabalho muito amigável, com facilidades para a modelagem, depuração, visualização da execução, análise estatística de resultados e geração de relatórios. Mas, sem dúvida os mais procurados atualmente são os recursos de animação, que vão desde simples implementações até recursos de animações em 3-D que, obviamente, consomem considerável espaço de memória e encarecem o produto.

Um procedimento de um estudo de simulação é ilustrado na Figura 3.

Figura 3 - Procedimento aplicado em um estudo de simulação

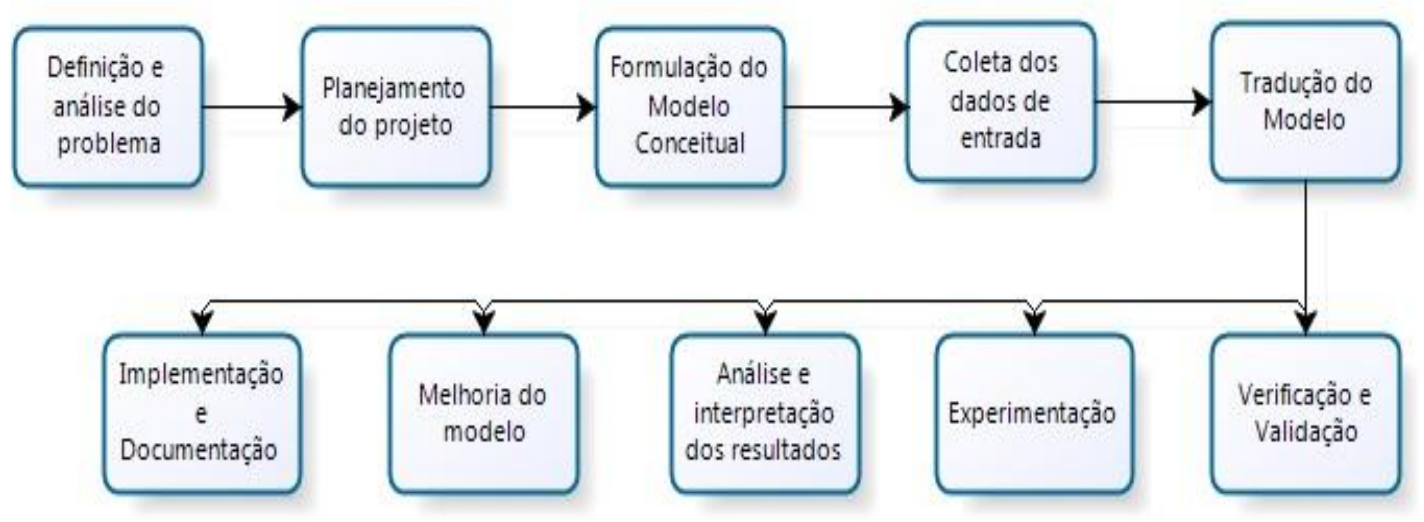

Fonte: Adaptado de [2]. 


\section{ESTUDO DA MOVIMENTAÇÃO DE NAVIOS NO PORTO DE ITAJAÍ}

Para apoiar a aplicação deste estudo de caso foram utilizadas a teoria das filas e a simulação discreta, descritas nos itens a seguir.

\subsection{APLICANDO A TEORIA DAS FILAS}

Antes de aplicar as equações $1-7$, definidas anteriormente, discutiram-se o tamanho da amostra e os diferentes parâmetros que são considerados para o uso destas. Também, são definidas as regras de atendimento que determinam a ordem em que os navios serão atendidos e isto será realizado por ordem de chegada (o primeiro a chegar é o primeiro a ser atendido).

Aplicando a teoria de filas (M/M/c/o/FIFO) no cenário atual, composto inicialmente por dois berços, este problema foi solucionado com o apoio de planilhas eletrônicas e os principais resultados estão ilustrados na Tabela 1.

Tabela 1. Resultados obtidos pela aplicação de Teoria das Filas

\begin{tabular}{crrr}
\hline & 2 Berços & 3 Berços & 4 Berços \\
\hline Taxa de utilização & 0,5 & 0,333 & 0,25 \\
Tempo de navios da fila & 6,5664 & 0,8945 & 0,1339 \\
Tempo de navios no sistema & 26,271 & 20,599 & 19,838 \\
Número de navios no sistema & 1,3333 & 1,0454 & 1,0068 \\
Número de navios na fila & 0,3333 & 0,0454 & 0,0068 \\
\hline
\end{tabular}

Posteriormente este problema foi solucionado com 3 e 4 berços operantes, sendo observadas melhorias significativas no tempo de operação e com isso a redução dos custos. A Tabela 1 apresenta os resultados dos cálculos com a situação atual e com o funcionamento dos outros dois berços.

\subsection{UTILIZANDO O SOFTWARE ARENA PARA SIMULAR}

A ferramenta simulação tem encontrado crescente receptividade, tanto no meio acadêmico como empresarial, notadamente para aplicações em sistemas de manufatura, movimentação e armazenagem de materiais. Um pacote de simulação que será utilizado neste trabalho é o processo de modelagem usando o simulador Arena, o modelo é estruturado e codificado com base na linguagem de simulação SIMAN por meio da seleção dos módulos que contêm as características dos processos a serem modelados. Utilizando os modelos disponibilizados pelo Arena, o usuário pode extrair módulos, posicioná-los no modelo e parametrizá-los de acordo com as características observadas no sistema real.

As principais medidas de desempenho avaliadas foram relacionadas ao tempo médio de espera dos navios para atracamento no porto, à quantidade média de carga descarregada e a taxa de utilização dos berços ativos. Uma das principais vantagens do Arena, fator crítico da escolha do software para este estudo, é a utilização de dados estocásticos para a simulação. Desta forma, não são utilizados valores médios para os parâmetros no modelo, e sim distribuições estatísticas geradas a partir de uma coleção de dados sobre o parâmetro a ser inserido [4]. 
Foram utilizados dados de movimentação dos navios (datas e horários de chegada na barra, tempos de manobra de atracação, espera para poder operar nos berços, operação nos berços, tempos de espera para atracação e desatracação), referentes aos meses de janeiro à agosto de 2013, concedidos pelo Porto de Itajaí. Com estes dados, foi feita uma tentativa de representá-los através de distribuições estatísticas, procurou-se verificar quais distribuições estatísticas mais se adequam aos dados dos intervalos acima mencionados.

Os dados fornecidos pelo Porto de Itajaí contêm, hora e data da chegada do navio na barra, tempo de espera na barra, tempo de manobra de atracamento, tempo de espera para o navio entrar em operação, tempo de operação, tempo de espera para o desatracamento (parte burocrática) e o tempo de desatracamento nos meses de abril a agosto de 2013 dos dois berços em funcionamento. As informações reais da movimentação dos navios foram primeiramente analisadas. $\mathrm{O}$ navio fica no sistema até o desatracamento em média 21,5 horas, desvio padrão de 11,3 horas.

Para isso, dados foram analisados através do software Arena (ferramenta Input Analyser), que permite realizar uma avaliação dos dados fornecidos, agrupando-os em intervalos de acordo com a distribuição estatística escolhida, indicando o valor da probabilidade destes dados ajustarem-se à função considerada. Através dessa avaliação, o software Arena gera um gráfico com a distribuição selecionada e apresenta resultados como: média da distribuição estatística, o valor do teste Qui-Quadrado, o número de graus de liberdade, o número de intervalos agrupados pelo programa, o valor da probabilidade de os dados aderirem a esta distribuição.

Desta forma, os dados de chegada do navio na barra, foram ajustados à distribuição que melhor as representará. A Figura 4 ilustra a modelagem destes dados e a função de distribuição de probabilidades Gamma.

Figura 4 - Distribuição Gamma gerado pelo software ARENA

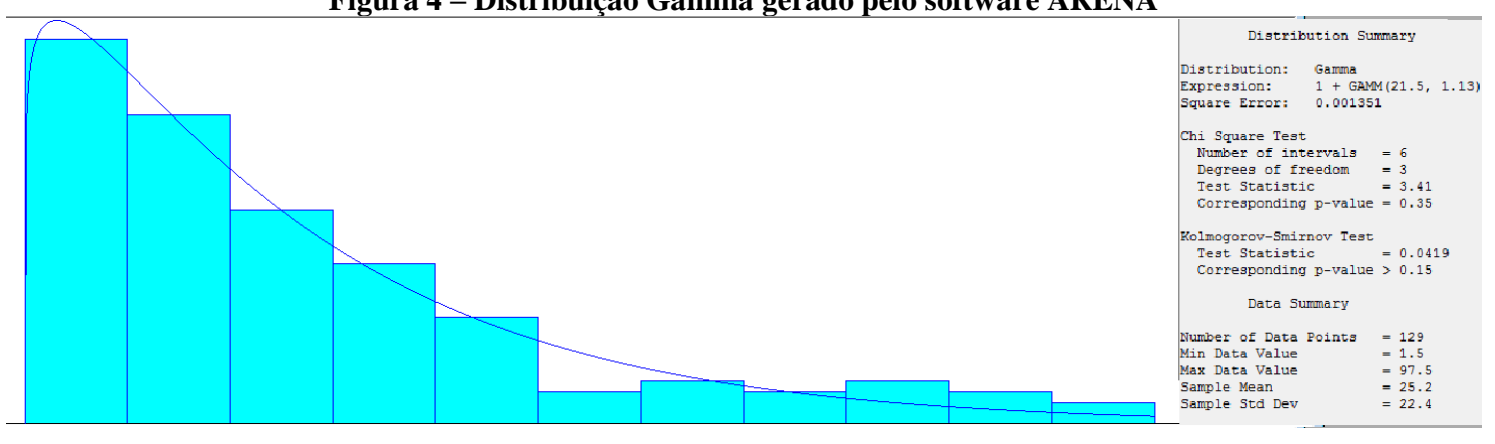

$1+\operatorname{GAMM}(21.5,1.13)$

Após a chegada, os navios esperam um tempo na barra para poder iniciar o processo de atracamento. Este processo é definido pela distribuição de lognormal conforme ilustra a Figura 5. 


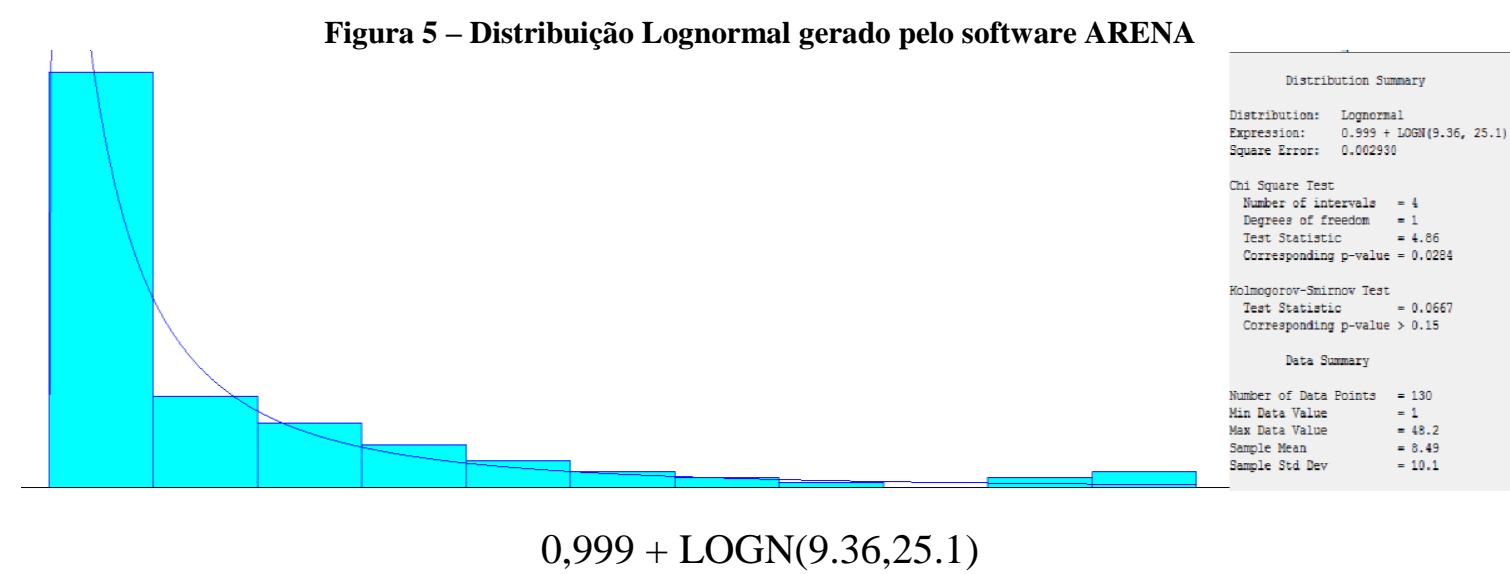

Da mesma forma, encontra-se o tempo da operação, que considera o tempo descarregamento e/ou carregamento. A Figura 6 ilustra a função distribuição Weibull, como a definida pela ferramenta.

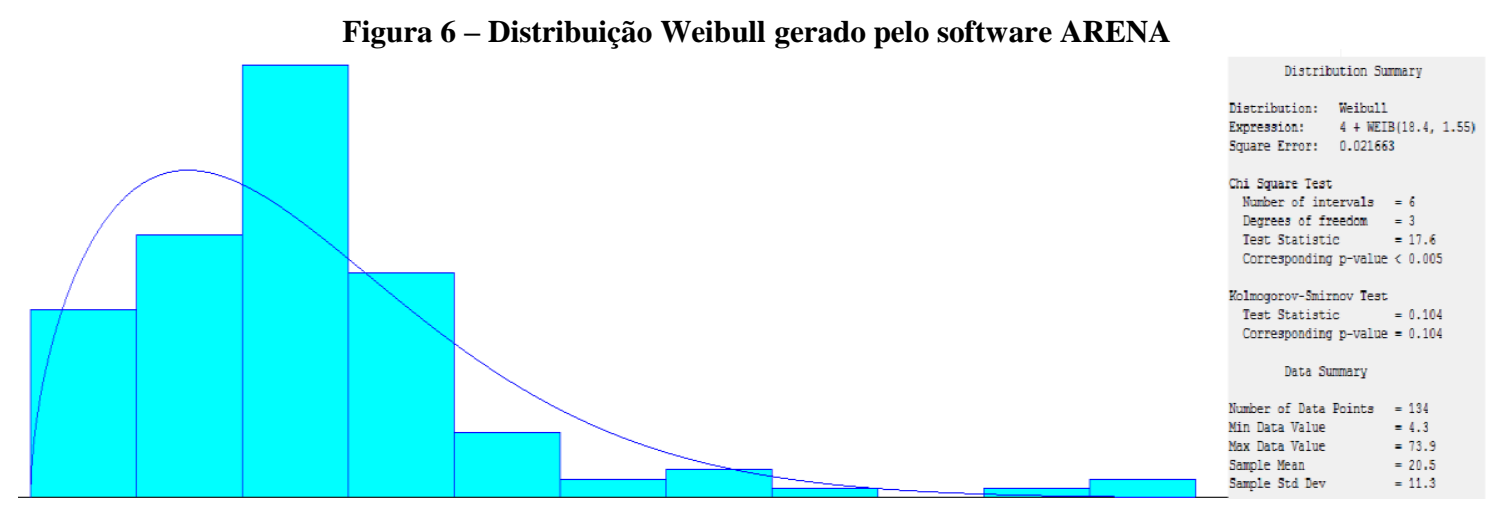

4 + WEIB(18.4,1.55)

Seguidamente o modelo do processo de movimentação de navios no Porto de Itajaí foi estruturado. Para isto, foi adicionado um objeto para representar a chegada dos navios, seguido de um processo de espera na barra e de uma decisão para a liberação dos berços. Os berços 2 e 3 são os que atualmente estão em funcionamento e os berços 1 e 4 são os que estão desativados, havendo diferenciação das cores na estrutura para identificação. Logo após, a operação do processo de carregamento/descarregamento, consecutivamente da desatracação do navio e finalizando com sua saída. O processo ficou estabelecido conforme ilustra a Figura 7. 
Figura 7 - Estrutura do modelo

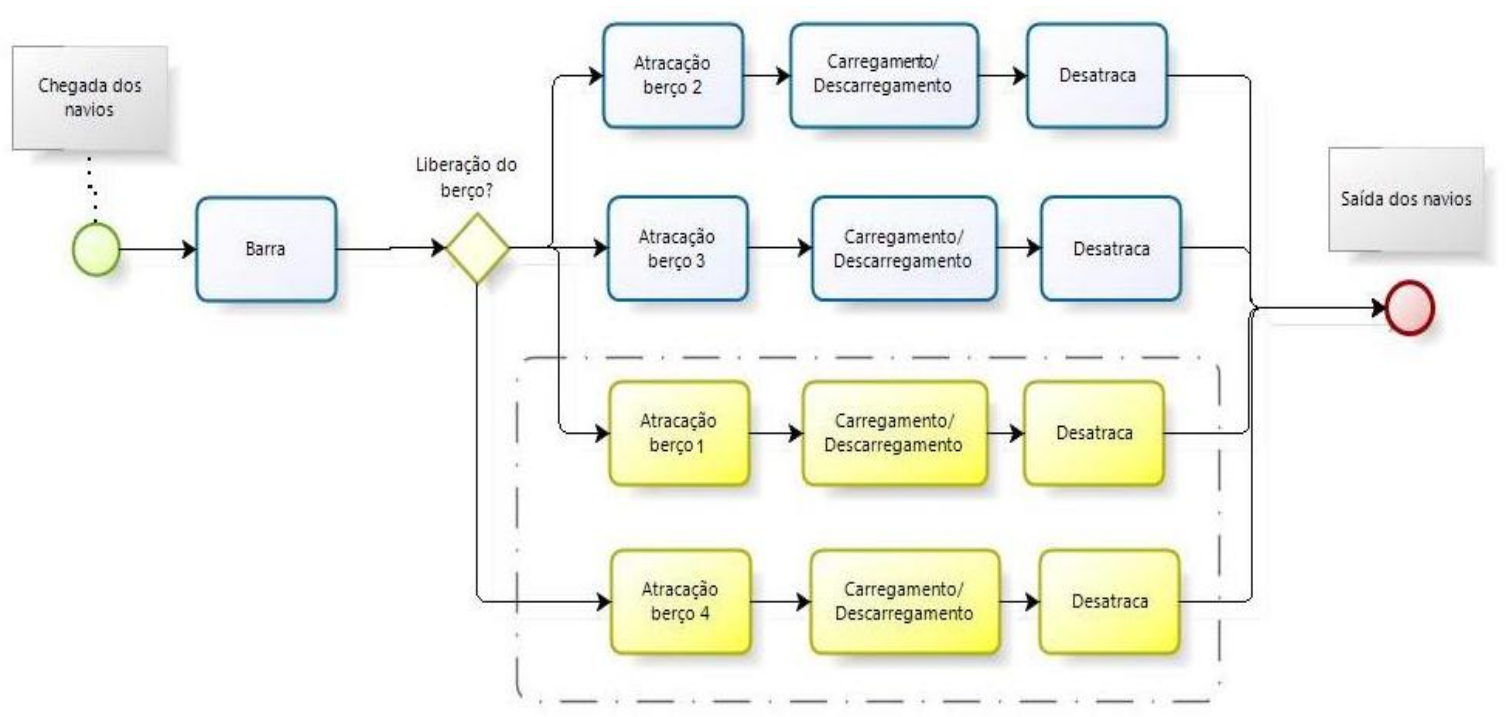

Fonte: Elaborado pelas Autoras.

Utilizando a ferramenta Arena foi realizada a simulação da movimentação dos navios no Porto de Itajaí e nesta foram consideradas as distribuições descritas anteriormente, assim como os berços disponíveis e os desativados do porto. Considerouse 5000 horas para a simulação. A tabela 2 fornece os resultados encontrados na simulação.

Tabela 2. Resultados obtidos através da simulação pelo Software ARENA

\begin{tabular}{crrr}
\hline & 2 Berços & 3 Berços & 4 Berços \\
\hline Taxa de utilização & 0,4632 & 0,3088 & 0,2316 \\
Tempo de navios da fila & 4,0038 & 0,534 & 0,0433 \\
Tempo de navios no sistema & 37,541 & 34,054 & 33,56 \\
Número de navios no sistema & 1,508 & 1,368 & 1,3488 \\
Número de navios na fila & 0,1609 & 0,0215 & 0,0017 \\
\hline
\end{tabular}

Comparando os resultados (Tabelas 1 e 2) das duas abordagens consideradas aqui (teoria das filas e simulação discreta), percebe-se que os resultados obtidos tiveram uma diferença considerável. Isso pode ser explicado pelo fato de a simulação na ferramenta Arena considerar o caráter aleatório das variáveis, fazendo uso de distribuições de probabilidade, na ocasião utilizada Lognormal, Weibull e Gamma, enquanto a teoria das filas se utiliza de valores constantes, apenas as médias das variáveis em seus cálculos, além das distribuições preestabelecidas, Exponencial e Poisson.

\section{CONCLUSÕES E CONSIDERAÇÕES FINAIS}

O Porto de Itajaí ocupa a terceira colocação no ranking nacional de exportações de contêineres. Suas instalações ocupam mais de $15 \mathrm{mil} \mathrm{m}^{2}$ de área coberta para estocagem de produtos e $38 \mathrm{mil} \mathrm{m}^{2}$ descobertos para armazenagem de contêineres. 
Atualmente, é constituído por quatro berços, sendo Berços 1 e 2 particulares e, os Berços 3 e 4 públicos. Devido a enchente em novembro de 2008 o Berço 4 foi danificado, impossibilitando a utilização até os tempos atuais. Já o berço 1 não está funcionando desde setembro de 2011, pelo mesmo motivo do berço 4 . Não há previsão para o reestabelecimento dos mesmos.

Uma contribuição deste trabalho foi avaliar um estudo sobre a importância dos dois berços desativados do Porto de Itajaí devido à enchente, mensurando em números os prejuízos causados não somente aos fornecedores, mas também aos clientes que esperam pelas suas mercadorias em seus destinos.

Normalmente certos conceitos relacionados ao fluxo do processo produtivo se tornam mais tangíveis quando visualizados na prática, pois apresentam algumas nuances que são compreendidas de forma mais clara quando vivenciadas em uma situação real. O presente artigo procurou demonstrar com dados reais e com o auxílio de um software capaz de simular situações comumente encontradas, como melhorar o atendimento no Porto de Itajaí a fim de que o tempo de espera no sistema diminua de forma com que os navios fiquem o menor tempo possível desde sua chegada à barra até sua desatracação do porto.

Com base nas análises e no modelo computacional criado, e atendendo aos objetivos desse estudo, conclui-se que a Teoria das Filas se aproximou mais da realidade. Nesse trabalho foi possível prever, de forma rápida, segura e sem custo algum, comportamentos futuros a partir de alterações feitas na quantidade de berços.

Com o funcionamento dos quatro berços o tempo de espera dos navios na fila e no sistema seria melhorado de forma relevante, aumentando a eficiência das operações portuárias e diminuindo consequentemente a probabilidade de um navio não achar um berço disponível.

Verifica-se que o Brasil ainda está longe de atingir os altos níveis de tecnologia e eficiência dos grandes portos mundiais. Uma perspectiva para pesquisa futura seria analisar a influência da parte operacional, como por exemplo, tempo de carregamento e descarregamento dos navios, e o impacto que isso gera no sistema portuário, visto que, há uma importância e uma grande exigência de um transporte marítimo moderno e eficiente, isto inclui os terminais e seus equipamentos de manuseio; e da parte burocrática, visto que hoje temos grandes atrasos na liberação de bens importados e exportados, em virtude da baixa capacidade operacional nestes setores, que são pecas fundamentais para a logística do processo.

\section{REFERÊNCIAS BIBLIOGRÁFICAS}

[1] ARENALES, M.; ARMENTANO, V.; MORABITO, R.; YANADASSE, H. Pesquisa Operacional. Rio de Janeiro: Elsevier, 2007.

[2] BANKS, J.; CARSEN, J. S. Discret Event System Simulation. Prentice-Hall, Englewood Cliffs, NJ, 1984.

[3] FAGERHOLT, K.; LINDSTAD, H. TurboRouter: An Interactive OptimisationBased Decision Support System for Ship Routing and Scheduling, Maritime Economics \& Logistics, 9, 214-233, 2007.

[4] FIORONI, M.M. Simulação em ciclo fechado de malhas ferroviárias e suas aplicações no brasil: avaliação de alternativas para o direcionamento de 
composições. Tese de Doutorado. Escola Politécnica da Universidade de São Paulo. São Paulo, 2007.

[5] FREITAS, P. J. Introdução à Modelagem e Simulação de Sistemas. Florianópolis - SC: Visual Books, 2010.

[6] Fogliatti, Mattos, FOGliatTi, M. C.; MATtOs, N. M. C. Teoria de filas. Rio de Janeiro: Interciência, 2007.

[7] HARREL, C. R.,MOTT, J. R. A., BATEMAN, R. E., BOWDEN, R. G. e GOGG, T. J. Simulação: otimizando os sistemas. Tradução de Alain de Norman et d'Audenhove. 2. ed. São Paulo: Imam, 2002.

[8] HILLIER, F.; LIEBERMAN, G. J. Introduction to Operations Research. 7. ed. São Paulo: McGraw-Hill, 2001.

[9] KELTON, D.; SADOWSKI, D. A.; SADOWSKI, R. P. Simulation with ARENA. New York, McGraw-Hill, 2010.

[10] PEGDEN, C. D., SHANNON, R. E., SADOWSKI, R. P. Introduction to Simulation using SIMAN, 2 ed, New York, McGraw-Hill Companies Inc. 1995.

[11] PRADO, D. S. Teoria das Filas e da Simulação. Nova Lima - MG: INDG Tecnologia e Serviços LTDA. 2006.

[12] PRADO, D. Usando o ARENA em Simulacão. Belo Horizonte: - MG: FALCONI, 2010.

[13] PORTOS DO BRASIL, disponível em: www.portosdobrasil.gov.br. Acessado em: $12 / 01 / 2015$.

[14] SALIBY, E. Softwares para Simulação. Rio de Janeiro, SOBRAPO - Pesquisa Operacional. V. 16, n. 1, 1996.

[15] SILVA, V. M. SOUZA, R. A., et al. Teoria das filas aplicada ao caso: Porto de Itajaí-SC. XIII SIMPEP - Bauru, SP, 2006.

[16] NORSTAD, I., FAGERHOLT, K. e LAPORTE, G. Tramp ship routing and scheduling with speed optimization, Transport Research, v. 19, p. $853-865$. 2010 .

[17] TECON, Publicação Técnica do Terminal de Contêineres, Santos, 2002. 